\title{
PERTURBATION OF A SURJECTIVE CONVOLUTION OPERATOR
}

\author{
I.KH. MUSIN
}

\begin{abstract}
Let $\mu \in \mathcal{E}^{\prime}\left(\mathbb{R}^{n}\right)$ be a compactly supported distribution such that its support is a convex set with a non-empty interior. Let $X_{2}$ be a convex domain in $\mathbb{R}^{n}, X_{1}=X_{2}+\operatorname{supp} \mu$. Let the convolution operator $A: \mathcal{E}\left(X_{1}\right) \rightarrow \mathcal{E}\left(X_{2}\right)$ acting by the rule $(A f)(x)=(\mu * f)(x)$ is surjective. We obtain a sufficient condition for a linear continuous operator $B: \mathcal{E}\left(X_{1}\right) \rightarrow$ $\mathcal{E}\left(X_{2}\right)$ ensuring the surjectivity of the operator $A+B$.
\end{abstract}

Keywords: convolution operator, distribution, Fourier-Laplace transform, entire functions.

Mathematics Subject Classification: 42B10, 44A35, 46E10

\section{INTRODUCTION}

1.1. On the problem and the main result. Given an open set $X$ in $\mathbb{R}^{n}$, by $\mathcal{E}(X)$ we denote the space of infinitely differentiable on $X$ functions with the topology defined by the system of the semi-norms

$$
\|f\|_{K, N}=\sup _{x \in K,|\alpha| \leqslant N}\left|\left(D^{\alpha} f\right)(x)\right|, K \Subset X, N \in \mathbb{Z}_{+} .
$$

The strongly dual space $\mathcal{E}^{*}(X)$ for the space $\mathcal{E}(X)$ consists of generalized functions compactly supported in $X$.

If $0 \neq \mu \in \mathcal{E}^{*}\left(\mathbb{R}^{n}\right), X_{1}, X_{2}$ are two non-empty open sets in $\mathbb{R}^{n}$ such that

$$
X_{2}+\operatorname{supp} \mu \subset X_{1}
$$

then the convolution $\mu * f$ of a distribution $\mu$ and the function $f \in \mathcal{E}\left(X_{1}\right)$ introduced by the rule

belongs to $\mathcal{E}\left(X_{2}\right)$.

$$
(\mu * f)(x)=\mu(f(x+y)), x \in X_{2},
$$

L. Ehrenpreis [1] and B. Malgrange [2] established that $P(D)\left(\mathcal{E}\left(\mathbb{R}^{n}\right)\right)=\mathcal{E}\left(\mathbb{R}^{n}\right)$ for each nonzero polynomial $P$ of $n$ variables. For a generalized function $\mu \in \mathcal{E}^{*}\left(\mathbb{R}^{n}\right), \mu \neq 0$, L. Ehrenpreis [3] proved that the convolution operator $f \rightarrow \mu * f$ acting from $\mathcal{E}\left(\mathbb{R}^{n}\right)$ into $\mathcal{E}\left(\mathbb{R}^{n}\right)$ is surjective if and only if $\mu$ is invertible, which means that its Fourier-Laplace transform $\hat{\mu}$ defined by the rule

$$
\hat{\mu}(z)=\mu\left(e^{\langle-i z, \xi\rangle}\right), z \in \mathbb{C}^{n}
$$

decays slowly, that is, there exists $a>0$ such that for each $\xi \in \mathbb{R}^{n}$ there exists a point $\eta \in \mathbb{R}^{n}$, for which $\|\xi-\eta\| \leqslant a \ln (2+\|\xi\|)$ and $|\hat{\mu}(\eta)| \geqslant(a+\|\xi\|)^{-a}$. In the general case, the problem on surjectivity of the convolution operator was given by L. Hörmander [4]-[6]. He proved that the convolution equation $\mu * f=g$ has a solution $f \in \mathcal{E}\left(X_{1}\right)$ for each $g \in \mathcal{E}\left(X_{2}\right)$ if and only if $\mu$

I.Kh. Musin, Perturbation of a surjective Convolution operator.

(c) Musin I.KH. 2016.

The work is supported by the Russian Foundation for Basic Researches (grant no. 15-01-01661) and the Program of the Presidium of RAS (project "Complex analysis and functional equations".)

Submitted June 25, 2016. 
is invertible and the pair $\left(X_{1}, X_{2}\right)$ is $\mu$-convex for the supports. We recall that a pair $\left(X_{1}, X_{2}\right)$ of open sets $X_{1}, X_{2}$ in $\mathbb{R}^{n}$ satisfying condition (1) is called $\mu$-convex for the supports [4, Def. 3.2], [5, Def. 3.2] if

$$
\operatorname{dist}\left(\operatorname{supp} \nu, \mathbb{R}^{n} \backslash X_{2}\right)=\operatorname{dist}\left(\operatorname{supp} \mu * \nu, \mathbb{R}^{n} \backslash X_{1}\right)
$$

for each $\nu \in \mathcal{E}^{*}\left(X_{2}\right)$. Here $\mu * \nu$ is the convolution of generalized functions $\mu$ and $\nu$ defined by the formula

$$
(\mu * \nu)(f)=\mu(\nu(f(x+y))), f \in \mathcal{E}\left(X_{1}\right),
$$

$\operatorname{dist}(A, B)=\inf \{\|x-y\|: x \in A, y \in B\},\|\cdot\|$ is the Euclidean norm in $\mathbb{R}^{n}$.

L. Hörmander showed [7, Thm. 5.4, Cor. 5.4] that if $\mu_{1}, \mu_{2} \in \mathcal{E}^{\prime}\left(\mathbb{R}^{n}\right)$ have disjoint singular supports and $\mu_{1}$ decays slowly, then $\mu_{1}+\mu_{2}$ decays slowly as well. Later the direct proof of this result by L. Hörmander was given by V. Abramchuk [8, Thm. 1]. Thus, if $\mu_{1}, \mu_{2} \in \mathcal{E}^{\prime}\left(\mathbb{R}^{n}\right)$ have disjoint singular supports and $\mu_{1}$ defines a surjective convolution operator $\mathcal{E}\left(\mathbb{R}^{n}\right)$, then the convolution operator associated with $\mu_{1}+\mu_{2}$ is also surjective.

Later there was not so many works devoted to the perturbations of the convolution operators in the spaces of infinitely differentiable functions. Among such works, we should mention rather recent results by C. Fernandez, A. Galbis and D. Jornet [9], who studied the behavior of the perturbations of the convolution operators in the space of ultra-differentiable functions in the sense of Braun, Meise and Taylor [10]. At that, the results of works [11], [12] on surjectivity of the convolution operators in these spaces were employed essentially.

In the present work the problem on surjectivity of the perturbed convolution operators is studied on the space of infinitely differentiable functions on convex domains in $\mathbb{R}^{n}$. The formulation of the problem differ from ones considered in [7], 8]. The formulation was essentially influenced by the studies by S.G. Merzlyakov [13] devoted to the perturbations of convolution operators in the spaces of holomorphic functions. Namely, let us fix a generalized function $\mu \in \mathcal{E}^{\prime}\left(\mathbb{R}^{n}\right)$, whose support is a convex set with a non-empty interior. Let $X_{2}$ be a convex domain in $\mathbb{R}^{n}$, $X_{1}=X_{2}+\operatorname{supp} \mu$. We note that in this case the pair $\left(X_{1}, X_{2}\right)$ is $\mu$-convex for the supports. This is implied by the theorem on the supports [6, Thm. 4.3.3] and the fact that for each convex domain $\Omega \subset \mathbb{R}^{n}$ and each compact set $K \subset \Omega$ we have $\operatorname{dist}\left(K, \mathbb{R}^{n} \backslash \Omega\right)=\operatorname{dist}\left(\operatorname{ch} K, \mathbb{R}^{n} \backslash \Omega\right)$. Here ch $K$ is the convex hull of the compact set $K$. Assume that the convolution operator $A: \mathcal{E}\left(X_{1}\right) \rightarrow \mathcal{E}\left(X_{2}\right)$ acting by the rule $(A f)(x)=(\mu * f)(x)$ is surjective (that is, $\mu$ is invertible). We consider the linear operator $B: \mathcal{E}\left(X_{1}\right) \rightarrow \mathcal{E}\left(X_{2}\right)$ such that for each compact set $K_{2}$ in $X_{2}$ there exist a convex compact subset $V$ in the interior of the support of $\mu$ (denoted by $\operatorname{supp} \mu$ ) and a number $N_{1} \in \mathbb{Z}_{+}$such that for each $\varepsilon>0$ less than the distance between $K_{2}$ and the boundary of $X_{2}$ and for each $N_{2} \in \mathbb{Z}_{+}$there exists a number $c=c\left(\varepsilon, N_{2}\right)>0$ such that

$$
\|B f\|_{K_{2}^{\varepsilon}, N_{2}} \leqslant c\|f\|_{K_{2}+V, N_{1}}, f \in \mathcal{E}\left(X_{1}\right) .
$$

Here $K_{2}^{\varepsilon}$ is an $\varepsilon$-swelling of the compact set $K_{2}$.

The main result of the work is the following theorem.

Theorem. The operator $A+B: \mathcal{E}\left(X_{1}\right) \rightarrow \mathcal{E}\left(X_{2}\right)$ is surjective.

1.2. Structure of the work. In Section 2 we provide two auxiliary results. The first is a principle of Phragmén-Lindelöf principle (Proposition 1). The second is the Hörmander division theorem [14, Cor. 2.6]. Here we also recall the definitions of two types of locally convex spaces in [15]. The main result is proved in Section 3. In Section 4 we provide an example of the operator $B$.

1.3. Some notations. Given $u=\left(u_{1}, \ldots, u_{n}\right) \in \mathbb{R}^{n}\left(\mathbb{C}^{n}\right), v=\left(v_{1}, \ldots, v_{n}\right) \in \mathbb{R}^{n}\left(\mathbb{C}^{n}\right)$, we let $\langle u, v\rangle=u_{1} v_{1}+\cdots+u_{n} v_{n}$ and $\|u\|$ stands for the Euclidean norm in $\mathbb{R}^{n}\left(\mathbb{C}^{n}\right)$.

For $\alpha=\left(\alpha_{1}, \ldots, \alpha_{n}\right) \in \mathbb{Z}^{n},|\alpha|=\alpha_{1}+\ldots+\alpha_{n}, D^{\alpha}$ is the corresponding partial derivative.

If $\Omega \subset \mathbb{R}^{n}$, then $\bar{\Omega}$, int $\Omega, \partial \Omega$, ch $\Omega$ is the closure, the interior, the boundary and the convex hull of $\Omega$, respectively. Given $\varepsilon>0$, we let $\Omega^{\varepsilon}=\left\{x \in \mathbb{R}^{n}:\|x-y\| \leqslant \varepsilon\right.$ for some $\left.y \in \Omega\right\}$. 
For $r>0$ we let $D(r)=\left\{x \in \mathbb{R}^{n}:\|x\|<r\right\}$.

The support function $H_{K}$ of a convex compact set $K \subset \mathbb{R}^{n}$ is introduced by the formula $H_{K}(y)=\max _{t \in K}\langle y, t\rangle, y \in \mathbb{R}^{n}$.

$H\left(\mathbb{C}^{n}\right)$ is the space of entire functions in $\mathbb{C}^{n}$.

The strongly dual space for a locally convex space $E$ is denoted by $E^{*}$.

\section{AuXiLiary INFORMATION AND RESUlts}

2.1. Auxiliary results. In the proof of Theorem the following two results will be useful.

Proposition 1. Let $b$ be a non-negative convex positive homogeneous of degree 1 function in $\mathbb{C}^{n}$ and $g \in H\left(\mathbb{C}^{n}\right)$. Assume that for each $\varepsilon>0$ there exists a constant $c_{\varepsilon}>0$ such that

$$
|g(z)| \leqslant c_{\varepsilon} \exp (b(z)+\varepsilon\|z\|), z \in \mathbb{C}^{n},
$$

and for some $M>0$ and $N \in \mathbb{Z}_{+}$

$$
|g(x)| \leqslant M(1+\|x\|)^{N}, x \in \mathbb{R}^{n} .
$$

Then

$$
|g(z)| \leqslant 2^{\frac{N}{2}} M(1+\|z\|)^{2 N} \exp (b(i \operatorname{Im} z)), z \in \mathbb{C}^{n} .
$$

This statement is an easy corollary of Lemma 1 given below and in fact, it was proved in [16]. In order to formulate it, we define a space $\mathcal{P}_{a}\left(T_{C}\right)$ as follows. Let $C$ be an open convex cone in $\mathbb{R}^{n}$ with the vertex at the origin and $a$ be a non-negative convex positive homogeneous of order 1 function in $\mathbb{R}^{n}+i \bar{C}$. Then $\mathcal{P}_{a}\left(T_{C}\right)$ is the space of function $f$ holomorphic in the tubular domain $T_{C}=\mathbb{R}^{n}+i C$ and satisfying the condition: for each $\varepsilon>0$ there exists a constant $c=c_{\varepsilon, f}>0$ such that

$$
|f(z)| \leqslant c \exp (a(z)+\varepsilon\|z\|), z \in \mathbb{R}^{n}+i C .
$$

Lemma 1. Let $g \in \mathcal{P}_{a}\left(T_{C}\right)$ and for $\xi \in \mathbb{R}^{n}$ we have $\varlimsup_{\substack{z \rightarrow \xi \\ z \in T_{C}}}|g(z)| \leqslant M$.

Then

$$
|g(x+i y)| \leqslant M \exp (a(i y)), x+i y \in T_{C} .
$$

Remark. It was assumed in [16, Lm.] that $C$ is an acute cone. The analysis of the proof of Lemma shows that this condition for $C$ is needless.

The following result was obtained by L. Hörmander [14, Cor. 2.6].

Proposition 2. For $j=1,2,3$ let $u_{j} \in \mathcal{E}^{\prime}\left(\mathbb{R}^{n}\right)$,

$$
H_{j}(\eta)=\sup \left\{\langle x, \eta\rangle, x \in \operatorname{supp} u_{j}\right\}
$$

and let $U_{j}$ be the Fourier-Laplace transform of $u_{j}$. Assume that $U_{2}=\frac{U_{3}}{U_{1}}$ is an entire function. Then $\mathrm{H}_{2}=\mathrm{H}_{3}-\mathrm{H}_{1}$ is the support function of some convex compact set in $\mathbb{R}^{n}$ and for each $\varepsilon>0$

where $C_{\varepsilon}>0$ is a constant.

$$
\left|U_{2}(\zeta)\right| \leqslant C_{\varepsilon} \exp \left(H_{2}(\operatorname{Im} \zeta)+\varepsilon\|\zeta\|\right), \quad \zeta \in \mathbb{C}^{n},
$$

2.2. Two definitions. We recall the definitions of $\left(M^{*}\right)$-space and $\left(L N^{*}\right)$-space in [15].

Definition 1. The space represented as the projective limit of the sequence of norm spaces $S_{n}, n \in \mathbb{N}$, w.r.t. linear continuous mappings $g_{m n}: S_{n} \rightarrow S_{m}, m<n$, such that $g_{n, n+1}$ are completely continuous for $n$ is called space $\left(M^{*}\right)$.

Definition 2. A locally convex space $E$ represented as an inductive limit of an increasing sequence of normed spaces $E_{k}$ such that the unit ball of the space $E_{k}$ is relatively compact in $E_{k+1}$ for each $k \in \mathbb{N}$ is called space $\left(L N^{*}\right)$. 
2.3. Some more notations and information. If $X$ is an open set in $\mathbb{R}^{n}$ and $\left(K_{m}\right)_{m=1}^{\infty}$ is a sequence of compact subsets in $X$ such that $K_{m} \subset$ int $K_{m+1}(m=1,2, \ldots)$ and $X=\cup_{k=1}^{\infty} K_{m}$, then by $C^{m}\left(K_{m}\right)$ we denote the normed space of functions $f$ smooth up to the order $m$ in $K_{m}$ with the norm $\|f\|_{K_{m}, m}$. We note that $\mathcal{E}(X)$ is a projective limit of the spaces $C^{m}\left(K_{m}\right)$. At that, $\mathcal{E}(X)$ is dense in each $C^{m}\left(K_{m}\right)$ and the embeddings $i_{m}: C^{m+1}\left(K_{m+1}\right) \rightarrow C^{m}\left(K_{m}\right)$ are completely continuous. Therefore, $\mathcal{E}(X)$ is an $\left(M^{*}\right)$-space. Then $\mathcal{E}^{*}(X)$ is an $\left(L N^{*}\right)$-space and $\mathcal{E}^{*}(\Omega)$ is the inductive limit of the spaces $\left(C^{m}\left(K_{m}\right)\right)^{*}[15$, Thm. 5].

\section{Proof OF THEOREM}

The theorem will be proved if we show that the image of the operator $A+B$ is closed and dense in $\mathcal{E}\left(X_{2}\right)$.

Let us show first that the image of the operator $A+B$ is closed in $\mathcal{E}\left(X_{2}\right)$. Since $\mathcal{E}\left(X_{1}\right)$ and $\mathcal{E}\left(X_{2}\right)$ are Frechét spaces, then the closedness of the operator $A+B$ is equivalent to the closedness of the image of the adjoint operator $(A+B)^{*}\left[17,8.6 .13\right.$, Thm.]. Since $\mathcal{E}^{*}\left(X_{1}\right)$ is an $\left(L N^{*}\right)$-space, to prove the closedness of the image of the operator $(A+B)^{*}$, it is sufficient to show that the image of the operator $(A+B)^{*}$ is sequentially closed [15, Prop. 8]. This is why assume that the functionals $S_{k} \in \mathcal{E}^{*}\left(X_{2}\right)$ are such that the sequence $\left((A+B)^{*} S_{k}\right)_{k=1}^{\infty}$ converges to $F \in \mathcal{E}^{*}\left(X_{1}\right)$ in $\mathcal{E}^{*}\left(X_{1}\right)$. For each $m \in \mathbb{N}$, let $X_{2, m}$ be its open bounded convex subset $X_{2}$ such that $\bar{X}_{2, m} \subset X_{2, m+1}, X_{2}=\cup_{m=1}^{\infty} \bar{X}_{2, m}$. We let $X_{1, m}=X_{2, m}+\operatorname{supp} \mu$. Then $\bar{X}_{1, m} \subset X_{1, m+1}, X_{1}=\cup_{m=1}^{\infty} \bar{X}_{1, m}$. By the properties of $\left(L N^{*}\right)$-spaces [15, Thm. 2, Cor. 1], there exists $p \in \mathbb{N}$ such that the functionals $F_{k}:=(A+B)^{*} S_{k}$ and $F$ belong to $\left(C^{p}\left(\bar{X}_{1, p}\right)\right)^{*}$ and the sequence $\left(F_{k}\right)_{k=1}^{\infty}$ converges to $F$ in $\left(C^{p}\left(\bar{X}_{1, p}\right)\right)^{*}$. Thus, the supports of the functionals $F_{k}$ and $F$ lie in $\bar{X}_{1, p}$ and the order of the distributions $F_{k}$ and $F$ does not exceed $p$.

Let $2 r_{p}:=\operatorname{dist}\left(\bar{X}_{2, p}, \partial X_{2, p+1}\right), \tilde{X}_{2}:=X_{2, p}+D\left(r_{p}\right)$ and $\tilde{X}_{1}:=\tilde{X}_{2}+\operatorname{supp} \mu$. We observe that $\tilde{X}_{1}$ and $\tilde{X}_{2}$ are bounded open convex sets in $\mathbb{R}^{n}$ an the pair $\left(\tilde{X}_{1}, \tilde{X}_{2}\right)$ is $\mu$-convex for the supports. We denote by $\tilde{A}$ the convolution operator $f \in \mathcal{E}\left(\tilde{X}_{1}\right) \rightarrow \mu * f$. It is obvious that $\tilde{A}$ acts from $\mathcal{E}\left(\tilde{X}_{1}\right)$ into $\mathcal{E}\left(\tilde{X}_{2}\right)$ linearly and continuously and if $f \in \mathcal{E}\left(X_{1}\right)$, then $\tilde{A} f=\tilde{A} f$. By the above cited result by L. Hörmander [5], $\tilde{A}\left(\mathcal{E}\left(\tilde{X}_{1}\right)\right)=\mathcal{E}\left(\tilde{X}_{2}\right)$.

Then, employing inequality (2), we continue uniquely the operator $B$ to a linear continuous operator $\tilde{B}$ acting from $\mathcal{E}\left(\tilde{X}_{1}\right)$ into $\mathcal{E}\left(\tilde{X}_{2}\right)$. We note that for each convex compact set $\tilde{K}_{2} \subset \tilde{X}_{2}$ there exist a compact $\operatorname{set} V \subset \operatorname{int}(\operatorname{supp} \mu)$ and a number $N_{1} \in \mathbb{Z}_{+}$such that for each $\varepsilon \in$ $\left(0, \operatorname{dist}\left(\tilde{K}_{2}, \partial \tilde{X}_{2}\right)\right)$ and for each $N_{2} \in \mathbb{Z}_{+}$there exists a number $c=c\left(\varepsilon, N_{2}\right)>0$ such that

$$
\|\tilde{B} f\|_{\tilde{K}_{2}^{\varepsilon}, N_{2}} \leqslant c\|f\|_{\tilde{K}_{2}+V, N_{1}}, f \in \mathcal{E}\left(\tilde{X}_{1}\right) .
$$

Letting $\tilde{K}_{2}=\overline{X_{2, p}}$, we confirm that $\tilde{B}$ is a compact operator from $\mathcal{E}\left(\tilde{X}_{1}\right)$ into $\mathcal{E}\left(\tilde{X}_{2}\right)$. By [17, Thm. 9.6.7], the image of the operator $\tilde{A}+\tilde{B}$ is closed in $\mathcal{E}\left(\tilde{X}_{2}\right)$. Therefore, the image of the operator $(\tilde{A}+\tilde{B})^{*}$ is closed in $\mathcal{E}^{*}\left(\tilde{X}_{1}\right)$.

Let $X_{2, j}=X_{2, p}+D\left(\frac{j}{j+1} r_{p}\right)$ for each $j \in \mathbb{N}$. Then $\tilde{X}_{1}=\cup_{j=1}^{\infty}\left(\tilde{X}_{2, j}+\operatorname{supp} \mu\right)$. We observe that for some $m \in \mathbb{N}$ the supports of the functionals $F, F_{k}=(A+B)^{*} S_{k}(k=1,2, \ldots)$ lie in $\overline{\tilde{X}}_{2, m}+\operatorname{supp} \mu$.

Mow we take an arbitrary functional $S_{k}$ and we are going to show that the convex hull $W_{k}$ of its support is located in $\overline{\tilde{X}}_{2, m+2}$. We suppose the opposite. Then there exists a point $\xi$ in $W_{k}$ not belonging to $\tilde{\tilde{X}}_{2, m+2}$. There exists a hyperplane in $\mathbb{R}^{n}$ separating $\overline{\tilde{X}}_{2, m+2}$ and $\xi$. This is why we can find a point $y_{0} \in \mathbb{R}^{n}$ such that

$$
H_{W_{k}}\left(y_{0}\right)>H_{\overline{\tilde{X}}_{2, m+2}}\left(y_{0}\right) .
$$


We denote the order of the distribution $S_{k}$ by $N_{2, k}$. We choose $\delta_{1}>0$ so small that $W_{k}^{\delta_{1}} \Subset X_{2}$. Then there exists a constant $a_{\delta_{1}, k}>0$ such that

$$
\left|\left(B^{*} S_{k}\right)(f)\right|=\left|S_{k}(B f)\right| \leqslant a_{\delta, k}\|B f\|_{W_{k}^{\delta_{1}}, N_{2, k}}, f \in \mathcal{E}\left(X_{1}\right) .
$$

By the assumption for $B$, there exists a convex compact set $V \subset \operatorname{int}(\operatorname{supp} \mu)$ and a number $N_{1} \in \mathbb{Z}_{+}$such that for the chosen $\delta_{1}>0$ there exists a constant $c_{\delta_{1}, k}>0$ such that

$$
\left|\left(B^{*} S_{k}\right)(f)\right| \leqslant c_{\delta_{1}, k}\|f\|_{W_{k}+V, N_{1}}, f \in \mathcal{E}\left(X_{1}\right) .
$$

For each $z \in \mathbb{C}^{n}$ this follows that

$$
\left.\mid \widehat{\left(B^{*} S_{k}\right.}\right)(z) \mid \leqslant c_{\delta_{1}, k}(1+\|z\|)^{N_{1}} \exp \left(H_{W_{k}}(\operatorname{Im} z)+H_{V}(\operatorname{Im} z)\right) .
$$

Taking into consideration that for some $d>0$

$$
H_{V}(x) \leqslant H_{\operatorname{supp} \mu}(x)-d\|\operatorname{Im} x\|, x \in \mathbb{R}^{n},
$$

by (4) we obtain that

$$
\left.\mid \widehat{\left(B^{*} S_{k}\right.}\right)(z) \mid \leqslant c_{\delta_{1}, k}(1+\|z\|)^{N_{1}} e^{H_{W_{k}}(\operatorname{Im} z)+H_{\text {supp }}(\operatorname{Im} z)-d\|\operatorname{Im} z\|}
$$

for all $z \in \mathbb{C}^{n}$. Since $F_{k} \in \mathcal{E}^{*}\left(X_{1}\right)$, supp $F_{k} \subset \overline{\tilde{X}}_{2, m}+\operatorname{supp} \mu$ and the order of the distribution $F_{k}$ does not exceed $p$, then for each $\delta>0$ there exists a constant $m_{\delta, k}>0$ such that

$$
\left|\hat{F}_{k}(z)\right| \leqslant m_{\delta, k}(1+\| z \mid)^{p} \exp \left(H_{\tilde{X}_{2, m}}(\operatorname{Im} z)+H_{\operatorname{supp} \mu}(\operatorname{Im} z)+\delta\|\operatorname{Im} z\|\right)
$$

for all $z \in \mathbb{C}^{n}$. Employing estimates (5) and (6) with $\delta=\frac{r_{p}}{2(m+1)(m+2)}$, we obtain that

$$
\left.\mid \widehat{\left(A^{*} S_{k}\right.}\right)(z) \mid \leqslant a(1+\| z \mid)^{b} e^{H_{\operatorname{ch}\left(W_{k} \cup \bar{X}_{2, m+1}\right)+\operatorname{supp} \mu}(\operatorname{Im} z)-\gamma\|\operatorname{Im} z\|}
$$

for all $z \in \mathbb{C}^{n}$, where $\gamma=\min (d, \delta), a=\max \left(c_{\delta_{1}, k}, m_{\delta, k}\right)$ and $b=\max \left(p, N_{1}\right)$. We choose a number $\gamma_{1} \in(0, \gamma)$ and we find a convex compact set $\Omega_{k} \subset \operatorname{int}\left(\operatorname{ch}\left(W_{k} \cup \tilde{\tilde{X}}_{2, m+1}\right)\right)$ such that

$$
H_{\mathrm{ch}\left(W_{k} \cup \tilde{X}_{2, m+1}\right)}(y)-H_{\Omega_{k}}(y) \leqslant \gamma_{1}\|y\|, y \in \mathbb{R}^{n} .
$$

Then by (7) we have

$$
\left.\mid \widehat{\left(A^{*} S_{k}\right.}\right)(z) \mid \leqslant a(1+|| z \mid)^{b} e^{H_{\Omega_{k}+\operatorname{supp} \mu}(\operatorname{Im} z)}
$$

We note that by Paley-Wiener-Schwartz theorem [6, Thm. 7.3.1] this means that the support of $A^{*} S_{k}$ is contained in $\Omega_{k}+\operatorname{supp} \mu$. Taking into consideration the identity

$$
\left.\widehat{\left(A^{*} S_{k}\right.}\right)(z)=\hat{S}_{k}(z) \hat{\mu}(z), z \in \mathbb{C}^{n}
$$

and Proposition 2, we obtain that $H_{\operatorname{supp}\left(A^{*} S_{k}\right)}-H_{\operatorname{supp} \mu}$ is the support function of some convex compact set $G_{k} \subset \mathbb{R}^{n}$ and for each $\varepsilon>0$ there exists a number $C_{\varepsilon}>0$ such that

$$
\left|\hat{S}_{k}(z)\right| \leqslant C_{\varepsilon} \exp \left(H_{G_{k}}(\operatorname{Im} z)+\varepsilon\|z\|\right), z \in \mathbb{C}^{n} .
$$

By Paley-Wiener-Schwartz theorem [6, Thm. 7.3.1], for some $M_{k}>0$ we have

$$
\left|\hat{S}_{k}(x)\right| \leqslant M_{k}(1+\|x\|)^{N_{2, k}}, x \in \mathbb{R}^{n} .
$$

Employing Proposition 1, by this inequality and (8) we obtain that

$$
\left|\hat{S}_{k}(z)\right| \leqslant M_{k}(1+\|z\|)^{2 N_{2, k}} e^{H_{G_{k}}(\text { Imz })}, z \in \mathbb{C}^{n} .
$$

Employing Paley-Wiener-Schwarz theorem [6, Thm. 7.3.1] once again, we obtain that the support of $S_{k}$ is contained in $G_{k}$. Therefore, for all $y \in \mathbb{R}^{n}$ we have

$$
H_{W_{k}}(y) \leqslant H_{G_{k}}(y)=H_{\operatorname{supp}\left(A^{*} S_{k}\right)}(y)-H_{\operatorname{supp} \mu}(y) \leqslant H_{\Omega_{k}+\operatorname{supp} \mu}(y)-H_{\operatorname{supp} \mu}(y)=H_{\Omega_{k}}(y) .
$$

In view of $\Omega_{k} \subset \operatorname{int}\left(\operatorname{ch}\left(W_{k} \cup \bar{X}_{2, m+1}\right)\right)$ this follows that

$$
H_{W_{k}}(y)<\max \left(H_{W_{k}}(y), H_{\tilde{X}_{2, m+1}}(y)\right), y \in \mathbb{R}^{n} .
$$


But this is impossible due to (3). Thus, for each $k \in \mathbb{N}$, the convex hull $W_{k}$ of the support of the functional $S_{k}(k=1,2, \ldots)$ is contained in $\overline{\tilde{X}}_{2, m+2}$.

Let $\eta \in \mathcal{E}\left(\mathbb{R}^{n}\right)$ be a function with a support in $\tilde{\tilde{X}}_{2, m+4}$ such that $0 \leqslant \eta(x) \leqslant 1$ for all $x \in \mathbb{R}^{n}$ and $\eta(x)=1$ for $x \in \overline{\tilde{X}}_{2, m+3}$. For each $k \in \mathbb{N}$ we define the functional $\tilde{S}_{k}$ on $\mathcal{E}\left(\tilde{X}_{2}\right)$ by the rule $\tilde{S}_{k}(f)=S_{k}(\eta f), f \in \mathcal{E}\left(\tilde{X}_{2}\right)$. It is obvious that $\tilde{S}_{k} \in \mathcal{E}^{*}\left(\tilde{X}_{2}\right)$ and $\tilde{S}_{k}(f)=S_{k}(f), f \in \mathcal{E}\left(X_{2}\right)$. We note that since for each $f \in \mathcal{E}\left(X_{1}\right)(A+B)(f)=(\tilde{A}+\tilde{B})(f)$, then the functionals $(A+B)^{*} S_{k}$ and $(\tilde{A}+\tilde{B})^{*} \tilde{S}_{k}(k=1,2, \ldots)$ coincide on $\mathcal{E}\left(X_{1}\right)$. Taking into consideration that $\mathcal{E}\left(X_{1}\right)$ is dense in $\mathcal{E}\left(\tilde{X}_{1}\right)$, we obtain that $(\tilde{A}+\tilde{B})^{*} \tilde{S}_{k}$ is the unique continuation of the functional $(A+B)^{*} S_{k}$ on $\mathcal{E}\left(\tilde{X}_{1}\right)$.

Let us show that the functionals $(\tilde{A}+\tilde{B})^{*} \tilde{S}_{k}$ converge in $\mathcal{E}^{*}\left(\tilde{X}_{1}\right)$. We first note that the sequence $\left((\tilde{A}+\tilde{B})^{*} \tilde{S}_{k}\right)_{k=1}^{\infty}$ is fundamental in $\mathcal{E}^{*}\left(\tilde{X}_{1}\right)$. Indeed, let $\mathcal{B}$ be an arbitrary bounded domain in $\mathcal{E}\left(\tilde{X}_{1}\right)$ and

$$
\mathcal{B}^{\circ}=\left\{F \in \mathcal{E}^{*}\left(\tilde{X}_{1}\right):|F(f)| \leqslant 1 \forall f \in \mathcal{B}\right\}
$$

are its polar. We take a function $\omega \in \mathcal{E}\left(\mathbb{R}^{n}\right)$ with the support in $\overline{\tilde{X}}_{2, m+4}+\operatorname{supp} \mu$ such that $0 \leqslant \omega(x) \leqslant 1$ for all $x \in \mathbb{R}^{n}$ and $\omega(x)=1$ for $x \in \overline{\tilde{X}}_{2, m+3}+\operatorname{supp} \mu$. Since the supports of the functionals $\tilde{S}_{k}$ lie in $\overline{\tilde{X}}_{2, m+2}$, the supports of the functionals $(\tilde{A}+\tilde{B})^{*} \tilde{S}_{k}$ are contained in $\tilde{X}_{2, m+2}+\operatorname{supp} \mu$. This is why for each $f \in \mathcal{E}\left(\tilde{X}_{1}\right)$ and all $k, m \in \mathbb{N}$ we have that

$$
\left((\tilde{A}+\tilde{B})^{*} \tilde{S}_{k}\right)(f)-\left((\tilde{A}+\tilde{B})^{*} \tilde{S}_{m}\right)(f)=\left((\tilde{A}+\tilde{B})^{*} \tilde{S}_{k}\right)(\omega f)-\left((\tilde{A}+\tilde{B})^{*} \tilde{S}_{m}\right)(\omega f) .
$$

We can consider $\omega f$ as an element of $\mathcal{E}\left(X_{1}\right)$ by letting $(\omega f)(x)=0$ for $x \in X_{1} \backslash\left(\tilde{X}_{2, m+4}+\operatorname{supp} \mu\right)$. Then

$$
\left((\tilde{A}+\tilde{B})^{*} \tilde{S}_{k}\right)(f)-\left((\tilde{A}+\tilde{B})^{*} \tilde{S}_{m}\right)(f)=\left((A+B)^{*} S_{k}\right)(\omega f)-\left((A+B)^{*} S_{m}\right)(\omega f) .
$$

We note that the set $\omega \mathcal{B}=\{\omega f: f \in \mathcal{B}\}$ is bounded in $\mathcal{E}\left(X_{1}\right)$. Since the sequence $((A+$ $\left.B)^{*} S_{k}\right)_{k=1}^{\infty}$ converges in $\mathcal{E}^{*}\left(X_{1}\right)$, it is fundamental in $\mathcal{E}^{*}\left(X_{1}\right)$. This is why there exists $N \in \mathbb{N}$ such that for all natural numbers $k, m \geqslant N$ and $g \in \omega \mathcal{B}$ we have $\left|\left((A+B)^{*} S_{k}\right)(g)-\left((A+B)^{*} S_{m}\right)(g)\right| \leqslant$ 1. Therefore, for all natural numbers $k, m \geqslant N$ and $f \in \mathcal{B}$ we obtain

$$
\left|\left((\tilde{A}+\tilde{B})^{*} \tilde{S}_{k}\right)(f)-\left((\tilde{A}+\tilde{B})^{*} \tilde{S}_{m}\right)(f)\right| \leqslant 1 .
$$

This means that for all natural numbers $k, m \geqslant N$ and $f \in \mathcal{B}$ we get $(\tilde{A}+\tilde{B})^{*} \tilde{S}_{k}-\left((\tilde{A}+\tilde{B})^{*} \tilde{S}_{m} \in\right.$ $\mathcal{B}^{\circ}$. Thus, we have proved that the sequence $\left((\tilde{A}+\tilde{B})^{*} \tilde{S}_{k}\right)_{k=1}^{\infty}$ is fundamental in $\mathcal{E}^{*}\left(\tilde{X}_{1}\right)$. Finally, since $\mathcal{E}^{*}\left(\tilde{X}_{1}\right)$ is complete, we obtain that the sequence $\left((\tilde{A}+\tilde{B})^{*} \tilde{S}_{k}\right)_{k=1}^{\infty}$ converges to some $\mathcal{E}^{*}\left(\tilde{X}_{1}\right)$ in $\tilde{T} \in \mathcal{E}^{*}\left(\tilde{X}_{1}\right)$. But $(\tilde{A}+\tilde{B})^{*}\left(\mathcal{E}^{*}\left(\tilde{X}_{2}\right)\right)$ is closed in $\mathcal{E}^{*}\left(\tilde{X}_{1}\right)$. Therefore, there exists a functional $\tilde{S} \in \mathcal{E}^{*}\left(\tilde{X}_{2}\right)$ such that $\tilde{T}=(\tilde{A}+\tilde{B})^{*} \tilde{S}$. Let $S$ be the restriction of $\tilde{S}$ on $\mathcal{E}\left(X_{2}\right)$. Then for each $f \in \mathcal{E}\left(X_{1}\right)$ we have $\tilde{T}(f)=T(f)$. Indeed,

$$
\begin{aligned}
\tilde{T}(f) & =\lim _{k \rightarrow \infty}\left((\tilde{A}+\tilde{B})^{*}\left(\tilde{S}_{k}\right)\right)(f)=\lim _{k \rightarrow \infty} \tilde{S}_{k}((\tilde{A}+\tilde{B}) f)=\lim _{k \rightarrow \infty} \tilde{S}_{k}((A+B) f) \\
& =\lim _{k \rightarrow \infty} S_{k}((A+B) f)=\lim _{k \rightarrow \infty}\left((A+B)^{*} S_{k}\right)(f)=T(f) .
\end{aligned}
$$

Together with the following chain of the identities

$$
\begin{aligned}
\tilde{T}(f) & =\lim _{k \rightarrow \infty}\left((\tilde{A}+\tilde{B})^{*}\left(\tilde{S}_{k}\right)\right)(f)=\left((\tilde{A}+\tilde{B})^{*}(\tilde{S})\right)(f)=\tilde{S}((\tilde{A}+\tilde{B}) f) \\
& =\tilde{S}((A+B) f)=S((A+B) f)=\left((A+B)^{*} S\right)(f)
\end{aligned}
$$

this implies that $T=(A+B)^{*} S$. Thus, the image of the operator $(A+B)^{*}$ is closed in $\mathcal{E}^{*}\left(X_{1}\right)$. Therefore, the image of the operator $A+B$ is closed in $\mathcal{E}\left(X_{2}\right)$.

Now we are going to prove that the image of the operator $A+B$ is dense in $\mathcal{E}\left(X_{2}\right)$. This will be done once we prove that an arbitrary functional $S \in \mathcal{E}^{*}\left(X_{2}\right)$ such that $S((A+B) f)=0$ for all $f \in \mathcal{E}\left(X_{1}\right)$ is the zero functional. We assume the opposite. Then the support of $S$ is 
non-empty. Let $N$ be the order of the distribution $S$ and $\delta>0$ is so small that $(\operatorname{supp} S)^{\delta} \Subset X_{2}$. Then there exists a constant $c_{\delta}>0$ such that

$$
|S(g)| \leqslant c_{\delta}\|g\|_{(\operatorname{supp} S)^{\delta}, N}, g \in \mathcal{E}\left(X_{2}\right) .
$$

By inequality (2) this yields that there exists a convex compact set $V \subset \operatorname{int}(\operatorname{supp} \mu$ ), a number $N_{1} \in \mathbb{Z}_{+}$depending on $\operatorname{ch}(\operatorname{supp} S)$ and a constant $C_{\delta}>0$ such that for each $f \in \mathcal{E}\left(X_{1}\right)$

$$
\left|\left(B^{*} S\right)(f)\right| \leqslant C_{\delta}\|f\|_{\operatorname{ch}(\operatorname{supp} S)+V, N_{1}} .
$$

Therefore, the support of the functional $B^{*} S$ is contained in $\operatorname{ch}(\operatorname{supp} S)+V$. On the other hand, the identity $B^{*} S=-\mu * S$ and the theorem on supports [6, Thm. 4.3.3],

$$
\operatorname{ch}\left(\operatorname{supp} B^{*} S\right)=\operatorname{ch}(\operatorname{supp} S)+\operatorname{supp} \mu .
$$

Thus, $\operatorname{ch}(\operatorname{supp} S)+\operatorname{supp} \mu \subset \operatorname{ch}(\operatorname{supp} S)+V$. But this inclusion is impossible since the convex compact set $V$ is contained in the interior of the support of $\mu$. Therefore, the assumption that $S$ is a non-zero functional is wrong. Thus, $S=0$. This means that the image of $A+B$ is dense in $\mathcal{E}\left(X_{2}\right)$. This completes the proof of the theorem.

\section{EXAMPle OF OPERATOR $B$}

Let $\mu \in \mathcal{E}^{\prime}\left(\mathbb{R}^{n}\right)$ is the invertible distribution and $\operatorname{supp} \mu=\overline{D(1)}$. The distribution with such properties can be constructed, see, for instance [8, Thms. 1, 3, 4]. Let $X_{2}=D(1)$, $X_{1}=D(2)$, and $A: \mathcal{E}\left(X_{1}\right) \rightarrow \mathcal{E}\left(X_{2}\right)$ be the convolution operator acting by the rule $(A f)(x)=$ $(\mu * f)(x), x \in X_{1}$. We take the function $b \in \mathcal{E}\left(\mathbb{R}^{2 n}\right)$ with the support in $\overline{D\left(\frac{1}{4}\right)} \times \overline{D\left(\frac{1}{4}\right)}$. We define the operator $B: \mathcal{E}\left(X_{1}\right) \rightarrow \mathcal{E}\left(X_{2}\right)$ by the rule

$$
\begin{aligned}
& (B f)(x)=\int_{\mathbb{R}^{n}} b(x, \xi) f(x+\xi) d \xi, \quad\|x\| \leqslant \frac{1}{4}, \\
& (B f)(x)=0, \quad \frac{1}{4}<\|x\|<1 .
\end{aligned}
$$

Let $K$ be a convex compact set in $X_{2}$ and $\gamma:=\operatorname{dist}\left(K, \partial X_{2}\right)$. Let us show that there exists a convex compact set $V \subset \operatorname{int}(\operatorname{supp} \mu)$ such that for all $\varepsilon \in(0, \gamma)$ and $N_{2} \in \mathbb{Z}_{+}$there exists a constant $c=c\left(\varepsilon, N_{2}\right)>0$ such that

$$
\|B f\|_{K^{\varepsilon}, N_{2}} \leqslant c\|f\|_{K+V, 0}, f \in \mathcal{E}\left(X_{1}\right) .
$$

It is obvious that for each $\varepsilon \in(0, \gamma)$ and each $N_{2} \in \mathbb{Z}_{+}$there exists a constant $C>0$ depending on $b$ and $N_{2}$ such that for each $f \in \mathcal{E}\left(X_{1}\right)$ we have

$$
\|B f\|_{K^{\varepsilon}, N_{2}}=\|B f\|_{K^{\varepsilon} \cap \overline{D\left(\frac{1}{4}\right)}, N_{2}} \leqslant C_{1}\|f\|_{\left(K^{\varepsilon} \cap \overline{\left.D\left(\frac{1}{4}\right)\right)}+\overline{D\left(\frac{1}{4}\right), 0}\right.} .
$$

If $\gamma \in\left(0, \frac{3}{4}\right)$, by (9) we get

$$
\|B f\|_{K^{\varepsilon}, N_{2}} \leqslant C_{1}\|f\|_{K^{\gamma}+\overline{D\left(\frac{1}{4}\right), 0}}=C_{1}\|f\|_{K+\overline{D\left(\gamma+\frac{1}{4}\right), 0}}
$$

Therefore, in this case we can let $V=\overline{D\left(\gamma+\frac{1}{4}\right)}$. If $\gamma \in\left[\frac{3}{4}, 1\right]$, then $K \subset \overline{D\left(\frac{1}{4}\right)}$ and by (9) we obtain

$$
\|B f\|_{K^{\varepsilon}, N_{2}} \leqslant C_{1}\|f\|_{\overline{D\left(\frac{1}{2}\right), 0}} \leqslant C_{1}\|f\|_{K+\overline{D\left(\frac{3}{4}\right), 0}} .
$$

Thus, as $\gamma \in\left[\frac{3}{4}, 1\right]$, we can let $V=\overline{D\left(\frac{3}{4}\right)}$.

Thus, by Theorem the operator $A+B: \mathcal{E}\left(X_{1}\right) \rightarrow \mathcal{E}\left(X_{2}\right)$ is surjective. 


\section{BIBLIOGRAPHY}

1. L. Ehrenpreis. Solution of some problems of division, Part I. Division by a polynomial of derivation // Amer. J. Math. 76:4, 883-903 (1954).

2. B. Malgrange. Existence et approximation des solutions des équations aux dérivées partielles et des équations de convolution // Ann. Inst. Fourier (Grenoble). 6, 271-355 (1956).

3. L. Ehrenpreis. Solutions of some problems of division, Part IV. Invertible and elliptic operators // Amer. J. Math. 82:3, 522-588 (1960).

4. L. Hörmander. On the range of differential and convolution operators. Institute for Advanced Study, Princeton, New Jersey. (1961).

5. L. Hörmander On the range of convolution operators // Ann. Math. 76:1, 148-170 (1962).

6. L. Hörmander. The analysis of linear partial differential operators I, II. Springer, Berlin (2003, 2005).

7. L. Hörmander. Supports and singular supports of convolutions // Acta Math. 110:1, 279-302 (1963).

8. W. Abramczuk. A class of surjective convolution operators // Pacific J. Math. 110:1, 1-7 (1984).

9. C. Fernandez, A. Galbis, D. Jornet. Perturbations of surjective convolution operators // Proc. Amer. Math. Soc. 130:8, 2377-2381 (2002).

10. R. Braun, R. Meise, B.A. Taylor. Ultradifferentiable functions and Fourier analysis // Results Math. 17:3, 206-237 (1990).

11. J. Bonet, A. Galbis, R. Meise. On the range of convolution operators on non-quasianalytic ultradifferentiable functions // Studia Math. 126:2, 171-198 (1997).

12. R. Braun, R. Meise, D. Vogt. Existence of fundamental solutions and surjectivity of convolution operators on classes of ultradifferentiable functions // Proc. London Math. Soc. 61:2, 344-370 (1990).

13. S.G. Merzlyakov. Perturbations of linear operators in spaces of holomorphic functions // Matem. Sborn. 186:3, 103-130 (1995). [Sb. Math. 186:3, 409-434 (1995).]

14. L. Hörmander. Convolution equations in convex domains // Inventiones math. 4:5, 306-317 (1968).

15. J. Sebastian-e-Silva. On some classes of locally convex spaces important in applications // Matematika. Sbornik Perevodov. 1, 60-77 (1957). (in Russian).

16. I.Kh. Musin. On the Fourier-Laplace representation of analytic functions in tube domains // Collect. Math. 45:3, 301-308 (1994).

17. R.E. Edwards. Functional analysis. Theory and applications Holt Rinehart and Winston, New York (1965).

Il'dar Khamitovich Musin,

Institute of Mathematics, Ufa Scientific Center, RAS,

Chernyshevsky str. 112,

450077, Ufa, Russia

E-mail: musin_ildar@mail.ru 